Access this article online

Quick Response Code:

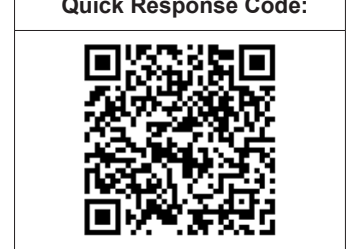

Website:

www.jlponline.org

DOI:

10.4103/JLP.JLP_44_16
Departments of

Pathology and

${ }^{1}$ Nephrology, JSS

Medical College, JSS

University, Mysore,

Karnataka, India

Address for correspondence:

Dr. Suchitha Satish,

\#892, I Block, $1^{\text {st }}$ Cross,

Ramakrishnanagar,

Mysore - 570 022,

Karnataka, India

E-mail: satishsuchitha@ gmail.com

Submission: 23-04-2016

Accepted: 23-01-2017

\title{
A clinico-pathological study of lupus nephritis based on the International Society of Nephrology-Renal Pathology Society 2003 classification system
}

\author{
Suchitha Satish, Pallavi Deka, Manjunath Sanjeev Shetty ${ }^{1}$
}

\section{Abstract:}

INTRODUCTION: Lupus nephritis (LN) is a major complication of systemic lupus erythematosus (SLE). Renal involvement is a major determinant of the prognosis of SLE. The histological classification of LN is a key factor in determining the renal survival of patients with LN. Prompt recognition and treatment of renal disease are important, as early response to therapy is correlated with better outcome and renal biopsy plays an important role in achieving this.

OBJECTIVES: The objective of this study was to correlate the clinical and laboratory findings with histopathological classes of LN as per the 2003 International Society of Nephrology-Renal Pathology Society (ISN/RPS) classification system.

PATIENTS AND METHODS: Fifty-six patients with SLE, undergoing a renal biopsy for renal dysfunction were studied. The comparison of data from multiple groups was made by Pearson's Chi-square test and between two groups by independent samples $t$-test. The values of $P<0.05$ were considered statistically significant.

RESULTS: Of the 56 cases studied, $51(91.1 \%)$ were females. The most common presenting symptoms were edema, arthralgia, and hypertension. Class IV (55.4\%) was the most common class. Thirty-nine (69.6\%) cases showed full house immunostaining. Hypertension, hematuria, proteinuria, and tubulo-interstitial disease showed a significant correlation $(P<0.05)$ with ISN/RPS classification, 2003.

CONCLUSION: Assessment and management of patients with suspected $L N$ are greatly facilitated through information obtained by renal biopsy. Since renal morphology may predict long-term prognosis, and no clinical or laboratory feature uniformly predicts prognosis, it is important to study the constellation of features in LN for better patient management.

Key words:

International Society of Nephrology-Renal Pathology Society classification, lupus nephritis, renal biopsy, systemic lupus erythematosus

\section{Introduction}

T upus nephritis (LN) is one of the most Lcommon manifestations of systemic lupus erythematosus (SLE), occurring in about $50 \%-70 \%$ of the patients, and is the major cause of morbidity and mortality in the SLE population. The clinical course of $\mathrm{LN}$ is heterogeneous and varies from subclinical disease to an aggressive course that may rapidly progress to end stage renal disease (ESRD). The nature and severity of the clinical features of LN do not always predict the underlying histological severity. Nevertheless, certain histological and clinical parameters have been associated with poor renal survival. ${ }^{[1]}$

This is an open access article distributed under the terms of the Creative Commons Attribution-NonCommercial-ShareAlike 3.0 License, which allows others to remix, tweak, and build upon the work non-commercially, as long as the author is credited and the new creations are licensed under the identical terms.

For reprints contact: reprints@medknow.com
Kidney biopsy is the main-stay for the diagnosis of LN. Material obtained by renal biopsy is evaluated by light microscopy (LM), immunofluorescence (IF), and electron microscopy. In many cases, renal biopsy is instrumental in establishing the diagnosis of $\mathrm{LN} .{ }^{[2]}$

The histopathological manifestations of LN are classified into several categories, originally designated by the WHO in 1982. These have recently evolved under the auspices of both the International Society of Nephrology and the Renal Pathology Society (ISN/RPS). ${ }^{[3]}$ The general structure includes six principal pathological patterns (Classes I-VI). ${ }^{[4]}$

\footnotetext{
How to cite this article: Satish S, Deka P, Shetty MS A clinico-pathological study of lupus nephritis based on the International Society of Nephrology-Renal Pathology Society 2003 classification system. J Lab Physicians 2017;9:149-55.
} 
Although clinical variables such as the elevation of serum creatinine, nephrotic syndrome at presentation, persistent elevations of blood pressure, low hematocrit, hypocomplementemia, and presence of anti-dsDNA antibodies have prognostic value, the histological information obtained from biopsies, the most important of which are the presence of crescents and interstitial fibrosis, continues to be indispensable in enhancing outcome prediction. ${ }^{[4]}$

Although certain histological and clinical parameters have been associated with poor renal survival, the results have been diverse due to difference in the population studied, sample size, selection criteria, and outcome variables chosen in different studies. ${ }^{[1]}$ It is therefore, relevant to document the clinico-pathological features of various patterns of renal diseases in SLE patients, in a south Indian tertiary care center.

\section{Patients and Methods}

This was a cross-sectional study conducted in the Departments of Pathology and Nephrology, JSS Medical college hospital, JSS University, Mysore for a period of $2 \frac{1}{2}$ years. A total of 56 patients were studied and included, undergoing a renal biopsy during the course of their clinical care as determined by their treating nephrologists. The definitive diagnosis of LN was based on clinical and laboratory findings and finally by LM and IF study. Ethical clearance from the Institutional Ethical Committee was obtained.

Data were collected as per the case report form. Preliminary investigations were performed as per the standard care at our institute. An informed consent before renal biopsy was taken from all patients. Renal biopsy was performed under local anesthesia using $2 \%$ lignocaine with an automated gun (Bard Company) and disposable needles. Two cores were taken. The tissue was placed in $10 \%$ formalin for light microscopic (LM) examination and in saline for IF studies. For LM, multiple step serials (32) from renal core were stained and studied using hematoxylin and eosin, periodic acid Schiff, Masson's trichrome stain, and Jones silver methanamine stain. For IF, the biopsy specimens were washed in phosphate-buffered saline (PBS) thrice followed by embedding the tissue for frozen section in optimum cutting temperature medium. Once the tissue was frozen, 2-3 micron thin sections were cut. One to two sections were layered on each slide and were labeled as IgG, IgA, IgM, C3, C1q, kappa, and lambda. The slides were then stained with fluorosceinisothiocynate labeled anti-human antibodies of IgG, IgA, IgM, C3, C1q, kappa, and lambda light chains (DACO), respectively. The slides were then incubated for an hour at $37^{\circ} \mathrm{C}$. After incubation the slides were again washed thrice with PBS, mounted with glycerin and viewed under immunofluorescent microscope-Olympus BX 41.

\section{Statistical evaluation}

The statistical analyses were performed using statistical package for social sciences (IBM SPSS Statistics for Windows, Version 21.0. Armonk, NY: IBM Corp) computer program. All continuous variables were expressed as mean \pm standard deviation and categorical variables were presented as percentage. The comparison of data from multiple groups was made by Pearson's Chi-square test and between two groups by independent samples $t$-test. $P<0.05$ were considered statistically significant. Graphs were generated using Microsoft excel 2013.

\section{Results}

During the study, a total of 56 patients with biopsy proven LN were reviewed. The baseline data at the time of renal biopsy is given in Table 1.

In this study, the age range was from 9 to 55 years with a mean age of $28.05 \pm 10.30$ years. The maximum number of cases was found to be in the range of 21-30 years. Fifty-one out of 56 patients $(91.1 \%)$ of the patients were females and five patients $(8.9 \%)$ were males with a male to female ratio of $1: 10.2$.

The most common clinical features consistent with SLE seen in this study were hematological abnormalities in $34(60.71 \%)$ (anemia [ $<11 \mathrm{~g} / \mathrm{dl}]$, leukopenia [<4,000 cells / cumm] or thrombocytopenia [ $<1$ lakh platelets/cumm]), followed by edema in $26(46.4 \%)$ and hypertension in $23(41 \%)$ patients. Skin lesions, malar rash, oral ulcers, joint pains suggestive of arthritis, pregnancy related complications in the form of history of recurrent pregnancy loss, anti-phospholipid antibody syndrome were seen and neurological disorder in the form of optic neuritis was noted. There was a statistically significant association $(P=0.007)$ of hypertension with ISN/RPS, 2003 classification of LN.

Classes of LN showed statistical significance with proteinuria and hematuria. No statistical significance was seen between classes of LN and anemia, serum creatinine or blood urea nitrogen [Table 2].

Renal function was categorized by the level of serum creatinine ( $\mathrm{mg} / \mathrm{dl}$ ) as follows: Normal-mild renal insufficiency $(<1.5)$, moderate renal insufficiency (1.5-3.0), and advanced renal insufficiency $(\geq 3.0)$. Renal insufficiency did not show any statistical significance $(P=0.095)$ with classes of LN [Table 3]. However, it showed a statistically significant correlation with hypertension $(P=0.015)$, with hypertensive patients having poorer renal function.

In this study, information on ANA was available for 55 patients of whom $54(98.18 \%)$ patients were positive. Anti-dsDNA was positive in $39(88.63 \%)$ patients in 44 patients for whom data were available.

Table 1: Baseline data at the time of renal biopsy

\begin{tabular}{lc}
\hline Parameters & $\begin{array}{c}\text { Baseline data at renal biopsy } \\
\text { (mean } \pm \text { SD) }\end{array}$ \\
\hline Age (years) & $28.05 \pm 10.30$ \\
Male to female ratio & $1: 10.2$ \\
Serum creatinine $(\mathrm{mg} / \mathrm{dl})$ & $1.79 \pm 1.271$ \\
Blood urea nitrogen $(\mathrm{mg} / \mathrm{dl})$ & $53.62 \pm 36.660$ \\
Urinary protein $(\mathrm{g} / \mathrm{L} / 24 \mathrm{~h})$ & $2.00 \pm 1.502$ \\
Serum albumin $(\mathrm{mg} / \mathrm{dl})$ & $2.56 \pm 0.512$ \\
Hemoglobin $(\mathrm{g} / \mathrm{dl})$ & $9.36 \pm 1.575$ \\
Activity index $(n=24)$ & $5.48 \pm 4.138$ \\
Chronicity index $(n=12)$ & $1.52 \pm 2.374$ \\
\hline
\end{tabular}

$\mathrm{SD}=$ Standard deviation 
Table 2: Renal function values in International Society of Nephrology-Renal Pathology Society classification of lupus nephritis, 2003

\begin{tabular}{lcccccc}
\hline \multirow{2}{*}{ Laboratory/clinical parameters } & \multicolumn{2}{c}{ Mean \pm SD } & \multicolumn{2}{c}{ Class VI } \\
\cline { 2 - 5 } & Class II & Class III & Class IV & Class V & \\
\hline Hemoglobin $(\mathrm{g} / \mathrm{dl})$ & $10.50 \pm 1.000$ & $9.50 \pm 1.958$ & $9.10 \pm 1.338$ & $9.67 \pm 1.751$ & 7.3 & 0.838 \\
Serum creatinine $(\mathrm{mg} / \mathrm{dl})$ & $1.00 \pm 0.000$ & $1.42 \pm 0.669$ & $2.08 \pm 1.440$ & $1.20 \pm 0.447$ & 5.3 & 0.176 \\
Blood urea nitrogen $(\mathrm{mg} / \mathrm{dl})$ & $19.00 \pm 1.414$ & $42.14 \pm 20.440$ & $61.67 \pm 38.558$ & $28.00 \pm 7.616$ & 123 & 0.78 \\
24 hours urine protein $(\mathrm{g} / \mathrm{L} / 24 \mathrm{~h})$ & $0.75 \pm 0.500$ & $1.22 \pm 1.202$ & $2.33 \pm 1.551$ & $2.25 \pm 1.500$ & 2.97 & 0.046 \\
Hematuria $(n=45), n(\%)$ & 0 & $5(11.1)$ & $16(35.6)$ & 0 & 0 \\
\hline
\end{tabular}

$\mathrm{SD}=$ Standard deviation

Renal biopsies from these patients were studied and on the basis of the histopathological and IF studies, were divided into the Classes I to VI according to the ISN/RPS, 2003 classification of LN [Figure 1]. Majority of the patients, that is, 31 (55.4\%) belonged to Class IV [Figure 2 and Table 4]. The class-wise distribution of various histopathological parameters is given in Table 5. Serum creatinine showed a statistical significance with tubular atrophy and interstitial fibrosis $(P=0.032)$ as well as tubulo-interstitial inflammation $(P=0.000)$.

Class IV was further divided into Class IV-S and IV-G. The differences between the two sub-groups are given in Table 6 .

Biopsies from all $56(100 \%)$ patients were positive for IgG. Biopsies from $43(76.7 \%)$ patients and $52(92.8 \%)$ patients were positive for IgA and IgM, respectively. Biopsies from $52(96.3 \%)$ patients were positive for complement C3 deposition and for C1q deposition, each.

Thirty-nine $(69.6 \%)$ biopsies showed full house staining with positivity for immunoglobulins IgG, IgA and IgM, and complements C3 and C1q [Figure 3].

Biopsies from 16 (28.5\%) patients showed tubulo-interstitial deposits on IF. All the 16 biopsies stained positively for IgG and nine $(56.25 \%)$ biopsies stained positively for C1q. Two $(3.57 \%)$ biopsies showed vascular immune deposits.

\section{Discussion}

SLE is an autoimmune disease characterized by chronic immune complex formation and variable manifestations that include multiple organ involvement, accompanied by multiple laboratory abnormalities, and frequent exacerbations. Renal involvement is common in SLE and often determines the course of the disease. Nearly $50-70 \%$ of all cases of SLE have some clinical manifestation of LN, mostly glomerulonephritis. ${ }^{[5]}$

In the present study, 51 out of 56 patients (91.1\%) were females with a male to female ratio of $1: 10.2$. This study shows a significant female predominance which is also seen in most of the studies. ${ }^{[1,5-11]} \mathrm{A}$ female phenotype is the major risk factor for the development of lupus. The female:male ratio increases from 2:1 in prepubertal children up to 4.5:1 in adolescence to the 8-12:1 reported in series of adult onset patients, falling back to $2: 1$ in patients over 60 years of age. These data are in accord with the theory that estrogens are precipitating factors in the emergence of lupus; whereas androgens are protective. ${ }^{[12]}$ The common clinical features seen in this study were consistent with other studies. ${ }^{[7,13-15]}$
Table 3: Renal insufficiency-class wise distribution

\begin{tabular}{|c|c|c|c|c|c|c|}
\hline \multirow{2}{*}{$\begin{array}{l}\text { Renal } \\
\text { insufficiency } \\
\text { (mg/dl) }\end{array}$} & \multicolumn{5}{|c|}{$n(\%)$} & \multirow[t]{2}{*}{$\boldsymbol{P}$} \\
\hline & Class II & Class III & Class IV & Class V & Class VI & \\
\hline $\begin{array}{l}<0.5 \\
\text { (normal-mild) }\end{array}$ & $4(100)$ & $8(66.67)$ & $13(50)$ & $4(80)$ & 0 & \multirow[t]{3}{*}{0.095} \\
\hline $\begin{array}{l}0.5-3 \\
\text { (moderate) }\end{array}$ & 0 & $4(33.33)$ & 7 (26.9) & $1(20)$ & 0 & \\
\hline$\geq 3$ (severe) & 0 & 0 & $6(23.1)$ & 0 & $1(100)$ & \\
\hline
\end{tabular}

Table 4: International Society of Nephrology-Renal Pathology Society Classification, 2003-class distribution of patients with lupus nephritis

\begin{tabular}{lc}
\hline Class of LN & Frequency (\%) \\
\hline Class I & 0 \\
Class II & $4(7.1)$ \\
Class III & $13(23.2)$ \\
Class IV & $31(55.4)$ \\
Class V & $7(12.5)$ \\
Class VI & $1(1.8)$ \\
\hline
\end{tabular}

$\mathrm{LN}=$ Lupus nephritis

In the present study, one patient had optic neuritis. Optic neuritis is uncommon in SLE, but may be the presenting feature of the disease. Ophthalmological and central nervous system manifestations occur in $20 \%-40 \%$ of patients with SLE. Involvement of the optic nerve or chiasm is rare and occurs in only $1 \%$ of patients with SLE. It is important to differentiate SLE associated optic neuritis from idiopathic optic neuritis because of the severe visual impairment and steroid dependence associated with the former. Early diagnosis and prompt treatment are important for restoring visual function in these patients. ${ }^{[16]}$

Hypertension was the most common complication encountered in this study with a significant correlation with renal insufficiency and classes of LN. Studies have shown that hypertension was associated with a poor renal function and poor outcome both at biopsy and follow-up ${ }^{[17]}$ and was found to be a poor prognostic factor. ${ }^{[17,18]}$ Furthermore, majority of patients with Class IV LN had hypertension, which corroborates with previous studies which show that hypertension is an added insult on top of severe glomerular inflammation in proliferative LN and may hasten the disease progression. ${ }^{[1,19]}$ These findings highlight the need for effectively treating this risk factor.

Proteinuria is one of the dominant signs of LN. ${ }^{[6]}$ There was a statistically significant association between proteinuria and classes of LN with patients with Class IV LN having 


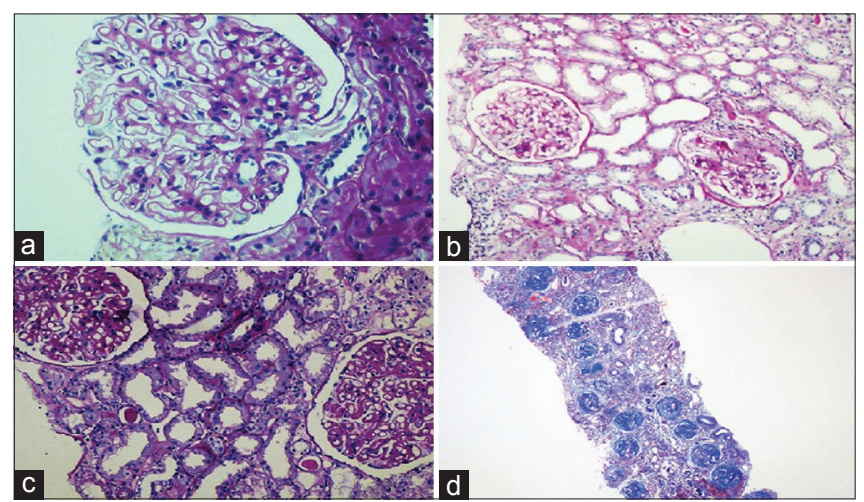

Figure 1: (a) Class II lupus nephritis - mesangial cell hypercellularity (periodic acid schiff $\times 200$ ). (b) Class III lupus nephritis - focal and segmental glomerulonephritis (periodic acid schiff $\times 100$ ). (c) Class V lupus nephritis thickened glomerular basement membrane (periodic acid schiff $\times 100$ ). (d) Class VI lupus nephritis - >90\% glomerular sclerosis (Masson's trichrome stain $\times 100$ )
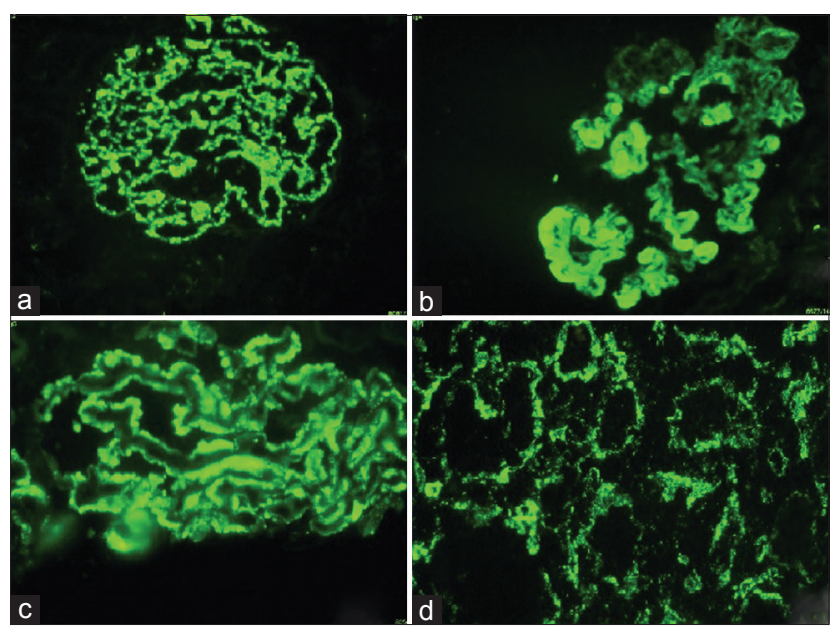

Figure 3: (a) Class IV lupus nephritis - mesangial and capillary loop deposits $(\operatorname{IgG} \times 100)$. (b) Class IV lupus nephritis - subendothelial deposits and hyaline globules $(\mathrm{IgG} \times 100)$. (c) Class V lupus nephritis - subepithelial deposits $(\lg \mathrm{x} \times 100)$. (d) Class IV lupus nephritis - tubulo-interstitial deposits $(\lg \mathrm{G} \times 100)$

predominantly nephrotic range of proteinuria. Previous studies have shown that nephrotic syndrome has a significant association with Class V LN, ${ }^{[5,14]}$ but in the present study, only one out of the four patients with Class V LN, presented with nephrotic syndrome. This could be attributed to the small number of patients with Class V LN in our study. Hematuria which showed a positive correlation with classes of LN was seen only in patients having proliferative LN (Class III and IV), a finding which is consistent with previous studies. ${ }^{[11]}$

An elevated serum creatinine level at the time of renal biopsy is associated with an increased rate of the development of renal failure and is a very strong adverse clinical prognostic indicator ${ }^{[5,17]}$ Serum creatinine showed a statistical significance with tubular atrophy and interstitial fibrosis as well as tubulo-interstitial inflammation, although no statistical significance was seen with classes of LN. This finding is in concordance with findings of previous studies which showed that interstitial infiltrates had prognostic value. ${ }^{[8,20,21]}$ Hill et al..$^{[8]}$ found interstitial inflammation to be one of the strongest histologic correlates of serum creatinine. ${ }^{[22]}$

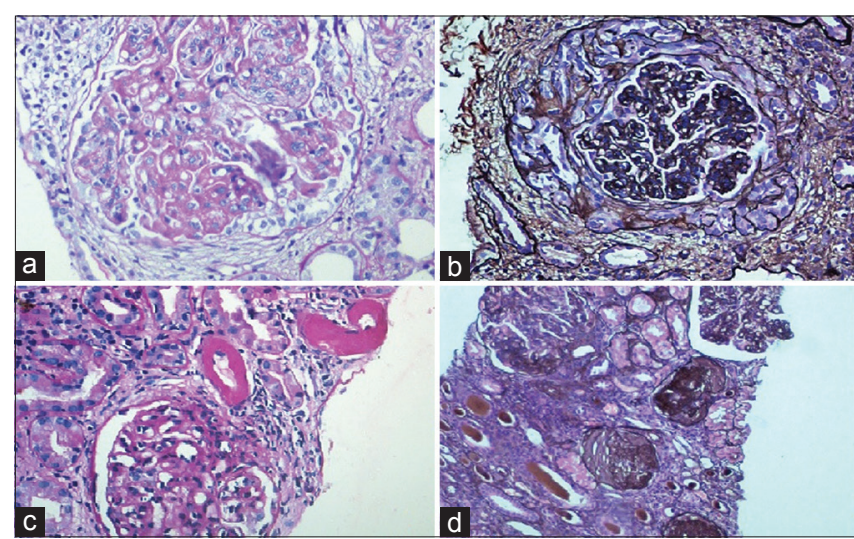

Figure 2: (a) Class IV lupus nephritis - endocapillary proliferation with wireloop lesions (periodic acid schiff $\times 200$ ). (b) Class IV lupus nephritis - circumferential cellular crescent (Jones silver methanamine stain $\times 200$ ). (c) Class IV lupus nephritis - thrombotic microangiopathy with fibrinoid necrosis of the arterioles (periodic acid schiff $\times 100$ ). (d) Class IV G (A/C) lupus nephritis - two proliferative glomeruli and three sclerotic glomeruli (Jones silver methanamine stain $\times 100$ )

High serum creatinine and greater degree of proteinuria indicate either severe disease presentation or a delay in the diagnosis of the disease, further emphasizing that early diagnosis, and prompt and aggressive treatment of proliferative $\mathrm{LN}$ is essential to improve outcomes of LN. ${ }^{[1]}$

Tubulo-interstitial inflammation by itself, whether measured in its entirety or confined to areas without atrophy or fibrosis, correlates with both renal function at biopsy and with renal survival. Therefore, the severity of interstitial nephritis serves as an independent prognostic indicator. ${ }^{[2]}$

The vast majority of patients in this study presented with Class IV LN, as has been seen in other studies and up to $40 \%$ of the patients with diffuse proliferative glomerulonephritis die or lose their renal function within 5 years after diagnosis. ${ }^{[5]}$ Because of its relative severity, this is the most important form of renal disease complicating SLE. ${ }^{[23]}$ This form of glomerular disease has a widely variable course ${ }^{[23-25]}$ and this has prompted a search for clinical and pathological factors that would enable better prognostication and management of the disease. ${ }^{[23-26]}$ In Class IV LN, the glomerular involvement is diffuse with either segmental or global involvement of the glomerular tuft. This subdivision comes in significant part as the result of a study by Najafi et al., ${ }^{[9]}$ in which it was found that cases with "severe segmental" glomerular lesions, involving $>50 \%$ of glomeruli (the equivalent of Class IV-S), had a worse outcome at 10 years than those with diffuse proliferative lesions involving the entire glomerular tuft (the equivalent of Class IV-G) $\cdot{ }^{[9,27]}$ In the present study, we did not find any significant difference $(P=0.228)$ between classes IV-S and IV-G in terms of the development of renal insufficiency. This is consistent with other studies that have found that there was no significant difference in survival and outcome between the patients with Class IV-S and IV-G lesions at the initial biopsy. ${ }^{[27-29]}$

Studies have shown that patients with LN classified as IV-G tend to have higher blood pressure, higher serum creatinine concentrations, more severe proteinuria, a higher level of anti-double-stranded DNA, lower hemoglobin levels and lower levels of C3 and C4 at baseline than do patients with 
Table 5: Class wise distribution of the various histological parameters

\begin{tabular}{|c|c|c|c|c|c|c|}
\hline Histopathology findings & Class I $(n=0)$ & Class II $(n=4)$ & Class III $(n=13)$ & Class IV $(n=31)$ & Class V $(n=7)$ & Class VI $(n=1)$ \\
\hline Mesangial hypercellularity (\%) & - & $4(100)$ & $13(100)$ & $31(100)$ & $7(100)$ & 0 \\
\hline Endocapillary hypercellularity (\%) & - & 0 & $13(100)$ & $31(100)$ & $3(42.9)$ & 0 \\
\hline Leucocyte infiltration (\%) & - & 0 & $10(76.9)$ & $29(93.5)$ & 0 & 0 \\
\hline Wireloop deposits (\%) & - & 0 & $2(15.4)$ & $20(64.5)$ & $2(28.6)$ & 0 \\
\hline Fibrinoid necrosis (\%) & - & 0 & 0 & $2(6.5)$ & 0 & 0 \\
\hline Karyorrhexis & & 0 & 0 & 0 & 0 & 0 \\
\hline Cellular cresents (\%) & - & 0 & $1(7.7)$ & $13(41.9)$ & 0 & 0 \\
\hline Interstitial inflammation (\%) & - & 0 & $5(38.5)$ & $25(80.6)$ & 0 & 0 \\
\hline Glomerular sclerosis (\%) & - & $1(25)$ & $6(42.9)$ & $11(35.5)$ & $4(57.1)$ & $1(100)$ \\
\hline Fibrous crescents (\%) & - & 0 & $2(15.4)$ & $3(9.7)$ & $1(14.3)$ & 0 \\
\hline Tubular atrophy (\%) & - & $1(25)$ & $2(15.4)$ & $11(35.5)$ & 0 & $1(100)$ \\
\hline Interstitial fibrosis (\%) & - & $1(25)$ & $2(15.4)$ & $11(35.5)$ & 0 & $1(100)$ \\
\hline BM spikes (\%) & - & 0 & 0 & 0 & $7(100)$ & 0 \\
\hline Activity index (mean $\pm S D)$ & - & 0 & $3.00 \pm 1.080$ & $8.13 \pm 3.442$ & $2.29 \pm 2.984$ & 0 \\
\hline Chronicity index $($ mean $\pm S D)$ & - & $0.50 \pm 1.000$ & $0.46 \pm 0.967$ & $1.26 \pm 1.932$ & $4.14 \pm 3.132$ & 9 \\
\hline
\end{tabular}

$\mathrm{SD}=$ Standard deviation, $\mathrm{BM}=$ Basement membrane spikes

Table 6: Class IV-segmental lupus nephritis versus Class IV-global lupus nephritis

\begin{tabular}{lccc}
\hline \multirow{2}{*}{$\begin{array}{l}\text { Laboratory/clinical } \\
\text { parameters }\end{array}$} & \multicolumn{2}{c}{ Mean \pm SD/\% } & $P$ \\
\cline { 2 - 3 } & Class IV-S $(n=3)$ & Class IV-G $(n=23)$ & \\
\hline Hemoglobin $(\mathrm{g} / \mathrm{dl})$ & $10.33 \pm 1.528$ & $8.89 \pm 1.231$ & $\mathrm{NS}$ \\
Serum creatinine $(\mathrm{mg} / \mathrm{dl})$ & $2.00 \pm 1.000$ & $2.14 \pm 1.558$ & $\mathrm{NS}$ \\
24 h proteinuria $(\mathrm{g} / \mathrm{L} / 24 \mathrm{~h})$ & $2.50 \pm 3.536$ & $2.28 \pm 1.432$ & 0.011 \\
Hematuria, $n(\%)$ & 0 & $16(76.2)$ & - \\
Hypertension, $n(\%)$ & $3(100)$ & $15(68.2)$ & - \\
Activity index & $4.67 \pm 2.082$ & $8.50 \pm 3.372$ & NS \\
Chronicity index & $2.00 \pm 3.464$ & $1.18 \pm 1.786$ & 0.048 \\
\hline
\end{tabular}

$\mathrm{LN}=$ Lupus nephritis, NS = Not significant, SD = Standard deviation, $\mathrm{S}=$ Segmental, $\mathrm{G}=$ Global

Class IV-S LN. However, these differences did not always reach statistical significance. ${ }^{[27,29]}$ The present study showed Class IV-G patients to have a lower mean hemoglobin and higher mean serum creatinine than Class IV-S patients although there was no statistical significance. Mean proteinuria in both groups were found to be similar, although it showed statistical significance with IV-S, which is inconsistent with previous studies. This could be explained by the small sample size of the Group IV-S.

The highly variable nature of LN has prompted investigation of prognostic features that would permit identification of those at high risk of renal failure. ${ }^{[2]}$ The clinical course of patients with diffuse proliferative glomerular lesions is probably influenced by the variable occurrence of certain histological features not specified by the conventional classification system of LN. Thus, detailed description of active and chronic, irreversible pathologic lesions affecting glomeruli, tubules, interstitium, and vasculature could augment the prognostic information derived from histologic classification and permit recognition of those patients with diffuse proliferative glomerulonephritis at high risk of ESRD. ${ }^{[24,30]}$

In the present study, the mean activity index was $5.48 \pm 4.138$ and the mean chronicity index was $1.52 \pm 2.374$. Chronic, sclerotic lesions in glomeruli are thought to be irreversible and refractory to therapy, whereas active lesions are reversible and can respond to therapy. Concordantly, persistent urinary protein was more often observed in the chronic types than in active types. Urinary protein by itself is an important risk factor for the deterioration of renal function, which is in part a result of direct interstitial inflammation. Such a feedback loop between sclerotic lesions and urinary protein can be a mechanism toward chronic renal injury. Even in very active cases of Class IV-G, the patients' renal outcome is favorable when the chronic lesions are absent, reiterating the importance of recording the activity and chronicity indices. ${ }^{[10]}$

It is thus recommended that patients with SLE have a kidney biopsy as soon as the clinical features of kidney involvement become evident, to hasten the treatment decisions and to minimize the risk of inflammation induced chronic kidney damage.

As in many other forms of glomerular disease, it is the distribution of deposits in the glomerular tuft that determines the proliferative response, and the glomerular lesions that result are therefore best interpreted in the context of the pattern of immune deposition. The IF staining pattern is often extremely helpful in confirming a diagnosis of LN when the diagnosis of SLE may be in doubt at the time of biopsy. ${ }^{[31]}$

The staining pattern is called "full house" when deposits containing all three immunoglobulin classes (IgG, IgM, and IgA) and both complement components (C3 and C1q) are present in LN. ${ }^{[31]}$ In the present study, 39 (69.6\%) of the biopsies showed full house staining.

Studies have shown that tubulo-interstitial immune deposits can be detected by fluorescence and electron microscopy in approximately $50 \%$ of patients. They occur more frequently in diffuse proliferative LN than in the focal proliferative variant, but they may also occur in some patients with membranous and mesangial proliferative forms. ${ }^{[31]}$ In the present study, $16(28.5 \%)$ biopsies showed tubulo-interstitial deposits on IF. Of the 16 biopsies, $13(81.25 \%)$ biopsies were from patients with Class IV LN, which is consistent with previous studies. ${ }^{[31]}$ The composition of the tubulo-interstitial deposits varies. Most have positive staining for IgG, with other immunoglobulins detected 
less frequently. ${ }^{[31]}$ Complement components are associated with the immunoglobulins in most cases and occasionally may be detected in their absence, ${ }^{[32]}$ suggesting a role for antibody-independent complement activation. ${ }^{[31]}$ In the present study, all the16 cases were positive for IgG and nine (56.25\%) biospies were positive for C1q.

\section{Conclusion}

Prompt recognition and treatment of renal disease in patients with SLE is important, as early response to therapy is correlated with better outcome. Renal biopsy provides information on the class of LN, tubular and vascular changes, activity, and chronicity indices. Class IV LN, by far has been seen to be the most important poor prognostic factor. Management goals in patients with LN include early diagnosis and appropriate therapy while preserving the overall kidney function without undue side-effects. This study which focuses on renal pathology will be an important addition to the existing literature and also provide information on the clinical utility of renal biopsy among SLE patients. Renal biopsy forms the cornerstone of management of patients with LN, as it provides invaluable information to the clinician, since approach to treatment of patients with different classes of LN is different.

\section{Acknowledgment}

We would like acknowledge Dr. Manjunath GV, Head of the Department of Pathology for his encouragement in carrying out this work.

Financial support and sponsorship Nil.

\section{Conflicts of interest}

There are no conflicts of interest.

\section{References}

1. Singh S, Zhou XJ, Ahn C, Saxena R. A retrospective analysis of clinical presentation of lupus nephritis. Am J Med Sci 2011;342:467-73.

2. Saxena R, Mahajan T, Mohan C. Lupus nephritis: Current update. Arthritis Res Ther 2011;13:240.

3. Weening JJ, D'Agati VD, Schwartz MM, Seshan SV, Alpers CE, Appel GB, et al. The classification of glomerulonephritis in systemic lupus erythematosus revisited. Kidney Int 2004;65:521-30.

4. Bihl GR, Petri M, Fine DM. Kidney biopsy in lupus nephritis: Look before you leap. Nephrol Dial Transplant 2006;21:1749-52.

5. Nezhad ST, Sepaskhah R. Correlation of clinical and pathological findings in patients with lupus nephritis: A five-year experience in Iran. Saudi J Kidney Dis Transpl 2008;19:32-40.

6. Brugos B, Kiss E, Szodoray P, Szegedi G, Zeher M. Retrospective analysis of patients with lupus nephritis: Data from a large clinical immunological center in Hungary. Scand J Immunol 2006;64:433-7.

7. Parichatikanond P, Francis ND, Malasit P, Laohapand T, Nimmannit S, Singchoovong L, et al. Lupus nephritis: Clinicopathological study of 162 cases in Thailand. J Clin Pathol 1986;39:160-6.

8. Hill GS, Delahousse M, Nochy D, Tomkiewicz E, Rémy P, Mignon F, et al. A new morphologic index for the evaluation of renal biopsies in lupus nephritis. Kidney Int 2000;58:1160-73.

9. Najafi CC, Korbet SM, Lewis EJ, Schwartz MM, Reichlin M,
Evans J; Lupus Nephritis Collaborative Study Group. Significance of histologic patterns of glomerular injury upon long-term prognosis in severe lupus glomerulonephritis. Kidney Int 2001;59:2156-63.

10. Hiramatsu N, Kuroiwa T, Ikeuchi H, Maeshima A, Kaneko Y, Hiromura K, et al. Revised classification of lupus nephritis is valuable in predicting renal outcome with an indication of the proportion of glomeruli affected by chronic lesions. Rheumatology (Oxford) 2008;47:702-7.

11. Yong JL, Killingsworth MC, Lai K. Renal biopsy pathology in a cohort of patients from Southwest Sydney with clinically diagnosed systemic lupus erythematosus. Int J Nephrol Renovasc Dis 2013;6:15-26.

12. Cameron JS. Lupus nephritis. J Am Soc Nephrol 1999;10:413-24.

13. Uramoto KM, Michet CJ Jr., Thumboo J, Sunku J, O'Fallon WM, Gabriel SE. Trends in the incidence and mortality of systemic lupus erythematosus, 1950-1992. Arthritis Rheum 1999;42:46-50.

14. Al-Zahrani IH, Qayyum A. Lupus nephritis. Clinicopathological correlation. Saudi Med J 2007;28:1503-5.

15. Tan Y, Song D, Wu LH, Yu F, Zhao MH. Serum levels and renal deposition of $\mathrm{C} 1 \mathrm{q}$ complement component and its antibodies reflect disease activity of lupus nephritis. BMC Nephrol 2013;14:63.

16. Lin YC, Wang AG, Yen MY. Systemic lupus erythematosus-associated optic neuritis: Clinical experience and literature review. Acta Ophthalmol 2009;87:204-10.

17. Halland AM, Bates WD, Tribe RD, Cooper R, Chalton D, Klemp P. Lupus nephritis. Part II. A clinicopathological correlation and study of outcome. S Afr Med J 1991;79:260-4.

18. Mok CC. Understanding lupus nephritis: Diagnosis, management, and treatment options. Int J Womens Health 2012;4:213-22.

19. Faurschou M, Dreyer L, Kamper AL, Starklint H, Jacobsen S. Long-term mortality and renal outcome in a cohort of 100 patients with lupus nephritis. Arthritis Care Res (Hoboken) 2010;62:873-80.

20. Park MH, D'Agati V, Appel GB, Pirani CL. Tubulointerstitial disease in lupus nephritis: Relationship to immune deposits, interstitial inflammation, glomerular changes, renal function, and prognosis. Nephron 1986;44:309-19.

21. Esdaile JM, Levinton C, Federgreen W, Hayslett JP, Kashgarian M. The clinical and renal biopsy predictors of long-term outcome in lupus nephritis: A study of 87 patients and review of the literature. Q J Med 1989;72:779-833.

22. Hsieh C, Chang A, Brandt D, Guttikonda R, Utset TO, Clark MR. Predicting outcomes of lupus nephritis with tubulointerstitial inflammation and scarring. Arthritis Care Res (Hoboken) 2011;63:865-74.

23. Magil AB, Puterman ML, Ballon HS, Chan V, Lirenman DS, Rae A, et al. Prognostic factors in diffuse proliferative lupus glomerulonephritis. Kidney Int 1988;34:511-7.

24. Austin HA $3^{\text {rd }}$, Muenz LR, Joyce KM, Antonovych TT, Balow JE. Diffuse proliferative lupus nephritis: Identification of specific pathologic features affecting renal outcome. Kidney Int 1984;25:689-95.

25. Magil AB, Ballon HS, Chan V, Lirenman DS, Rae A, Sutton RA. Diffuse proliferative lupus glomerulonephritis. Determination of prognostic significance of clinical, laboratory and pathologic factors. Medicine (Baltimore) 1984;63:210-20.

26. Austin HA $3^{\text {rd }}$, Muenz LR, Joyce KM, Antonovych TA, Kullick ME, Klippel JH, et al. Prognostic factors in lupus nephritis. Contribution of renal histologic data. Am J Med 1983;75:382-91.

27. Hill GS, Delahousse M, Nochy D, Bariéty J. Class IV-S versus class IV-G lupus nephritis: Clinical and morphologic differences suggesting different pathogenesis. Kidney Int 2005;68:2288-97.

28. Sada KE, Makino H. Usefulness of ISN/RPS classification of lupus nephritis. J Korean Med Sci 2009;24 Suppl: S7-10. 
29. Haring CM, Rietveld A, van den Brand JA, Berden JH. Segmental and global subclasses of class IV lupus nephritis have similar renal outcomes. J Am Soc Nephrol 2012;23:149-54.

30. Schwartz MM, Bernstein J, Hill GS, Holley K, Phillips EA. Predictive value of renal pathology in diffuse proliferative lupus glomerulonephritis. Lupus Nephritis Collaborative Study Group. Kidney Int 1989;36:891-6.

31. D'Agati VD, Stokes MB. Renal disease in systemic lupus erythematosus, mixed connective tissue disease, Sjögren syndrome, and rheumatoid arthritis. In: Jennette JC, Olson JL, Silva FG, D'Agati VD, editors. Hepinstall's Pathology of Kidney. $7^{\text {th }}$ ed. Philadelphia: Lippincott Williams and Wilkins; 2014.

32. Asherson RA, Hughes GR, Derksen RH. Renal infarction associated with anti-phospholipid antibodies in systemic lupus erythematosus and "lupus-like" disease. J Urol 1988;140:1028. 